Ann. Zootech., I966, 15 (4), 353-359.

\title{
INFLUENCE DE L'EXGITATION ANTE MORTEM CHEZ LE PORC SUR QUELQUES CARACTÉRISTIQUES PHYSICO-CHIMIQUES DU MUSCLE
}

\author{
J. CHARPENTIER et R. GOUTEFONGEA \\ avec la collaboration technique de J.-P. SuqueT \\ Laboratoire de Recherches sur la Viande, \\ Centre national de Recherches zootechniques, 78-Jouy-en-Josas,
}

SOMMAIRE

L'excitation provoquée par une série de décharges électriques à des temps variables avant l'abattage, entraîne, chez le porc, une augmentation du $\mathrm{pH}$, de la rétention d'eau et de la couleur du tissu musculaire. Un délai de 30 minutes entre l'excitation et l'abattage est néanmoins nécessaire pour que ces caractéristiques soient modifiées. Les conséquences de l'excitation sont moins importantes lorsque ce délai augmente. Cette expérience montre qu'une excitation d'intensité relativement faible et par ailleurs très fréquente lors du transport des animaux, peut avoir de notables répercussions sur la qualité de la viande.

De nombreux travaux montrent que, dans l'espèce porcine notamment, les excitations variées que les animaux sont susceptibles de subir avant 1'abattage peuvent modifier profondément certaines caractéristiques du tissu musculaire telles que le $\mathrm{pH}$, la couleur et la capacité de rétention d'eau (CALLOW, I936, I937, I938, I939; Henry et al., I958 ; Henry, I959 ; Wismer-Pedersen, I959 ; Wismer-PEDERSEN et Rieman, ig60 ; LEwis et al., I959, I962 ; SAYre et al., I96I, I963; BRISKey, ig64).

L'importance de ces modifications s'avère toutefois très variable. Il semble que la discordance entre certains résultats expérimentaux soit imputable aux variations dans les modalités mêmes des excitations et dans la durée du temps de repos avant l'abattage. Aussi nous a-t-il semblé intéressant d'étudier l'influence de ce dernier paramètre dans le cas d'excitations assez analogues à celles que les animaux peuvent effectivement subir lors de leur transport vers les centres d'abattage. 


\section{I. - MA'TÉRIEL ET MÉTHODES}

Les 64 porcs utilisés dans cette expérience provenaient du troupeau expérimental de la Station de Recherches sur l'Élevage des Porcs du C. N. R. Z. Les animaux furent abattus au poids de 100 $\pm 5 \mathrm{~kg}$. Comme les divers traitements furent appliqués à des dates différentes, lors de chaque abattage les animaux expérimentaux furent comparés au même nombre d'ánimaux témoins. Chaque traitement fut appliqué sur un lot de 8 animaux. Les différents traitements consistaient en une excitation électrique périodique effectuée :
a) pendant les 5 minutes précédant l'abattage .......
lot I
b) 30 minutes avant l'abattage $\ldots \ldots \ldots \ldots \ldots \ldots$ lot 2
c) 8 heures avant l'abattage $\ldots \ldots \ldots \ldots \ldots \ldots \ldots \ldots \ldots \ldots \ldots \ldots$ lot 3
d) 16 heures avant l'abattage $\ldots \ldots \ldots \ldots \ldots \ldots$ lot 4

L'excitation consistait en l'application de 20 décharges produites par un bâton électrique de 9 volts "Kawe " d'un type utilisé couramment lors du transport des animaux, à raison d'une décharge toutes les quinze secondes.

Les animaux furent abattus à l'aide d'un pistolet à broche "Matador ".

Les animaux témoins furent abattus dans leur loge en évitant soigneusement toute excitation préalable.

Quarante-cinq minutes après l'abattage, le $\mathrm{pH}$ fut déterminé à l'aide d'un $\mathrm{pH}$ mètre EIL sur les muscles suivants :

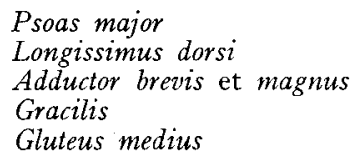

Les carcasses furent mises en chambre froide à $+4^{\circ} \mathrm{C}, 2$ heures après l'abattage. Le $\mathrm{pH}$ fut déterminé 24 heures après l'abattage aux mêmes sites des muscles précédents et également sur la partie du Biceps femoris apparaissant sur la coupe du jambon. Le pourcentage d'eau perdue par compression d'un échantillon de 5 grammes de broyat musculaire pendant $\mathrm{I}$ minute fut déterminé à l'aide d'un appareil spécial (GoUTEFONGEA, résultats non publiés) sur les muscles suivants :

\section{Psoas major \\ Gluteus medius \\ Biceps femoris}

L'intensité de la coloration fut déterminée sur ces mêmes muscles par le pourcentage de rémission à $490 \mathrm{~m} u$ obtenu à l'aide d'un réflectomètre électrosynthèse $\mathrm{SP}_{3}$.

\section{II. - RÉSULTATS. DISCUSSION}

\section{I. $p H$ " 45 minutes " et $p H$ " 24 heures"}

L Les valeurs moyennes des " $\mathrm{pH} 45$ " des différents muscles pour les quatre traitements et le lot témoin figurent dans le tableau I ainsi que les valeurs correspondantes du test $t$.

Il en résulte que le traitement $\mathbf{n}^{\circ}$ I n'exerce pas d'influence sur la vitesse de chute du $\mathrm{pH}$. Pour les autres traitements, la quasi-totalité des " $\mathrm{pH} 45$ " est supérieure à 6,00 . 

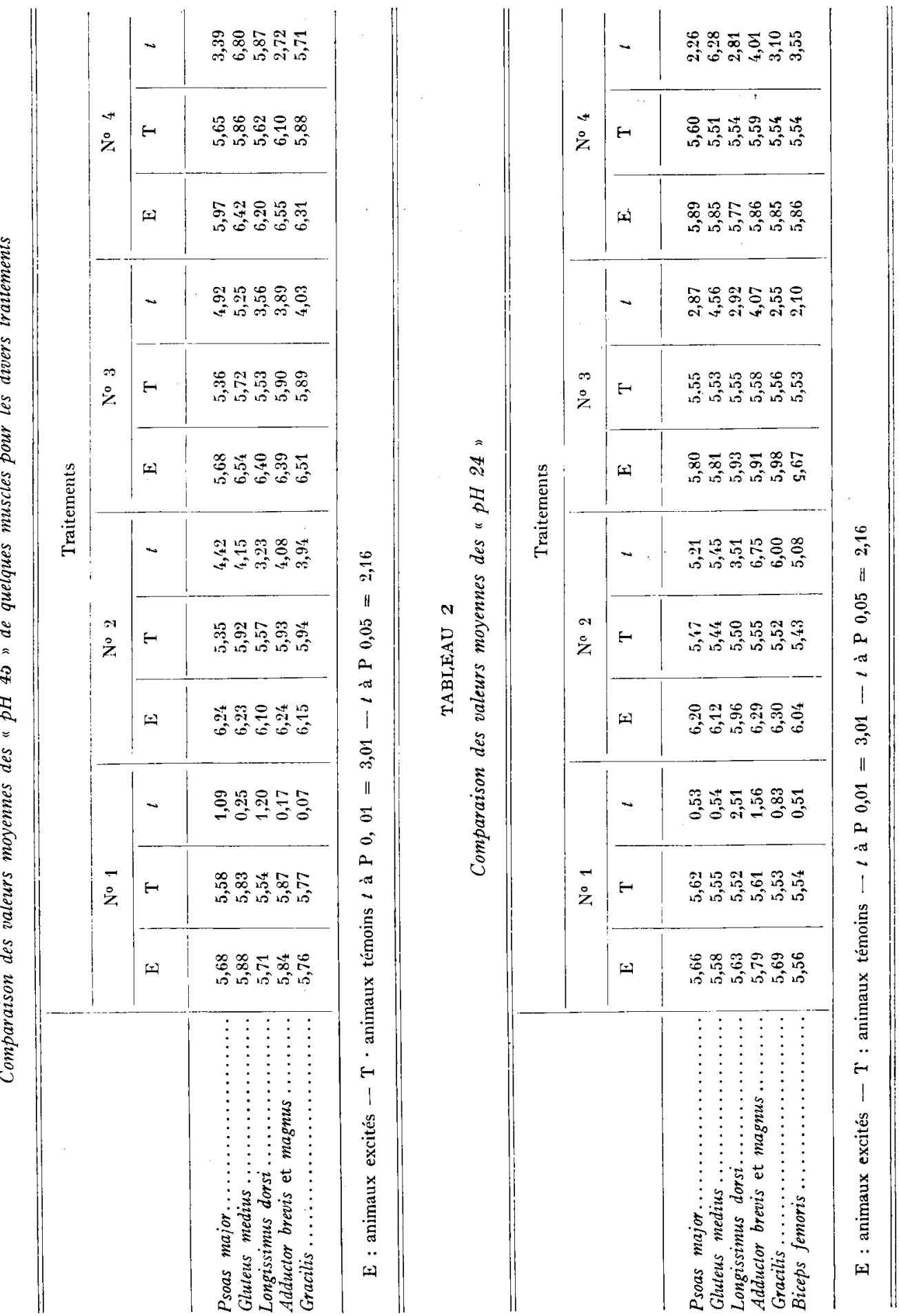
La comparaison des valeurs moyennes des $\mathrm{pH} 24$ heures (tabl. 2) montre que :

- le pH 24 n'est pas modifié significativement par le traitement $n^{0}$ I ;

- par contre, les autres traitements conduisent à des valeurs très différentes du $\mathrm{pH} 24$ entre les animaux excités et les animaux témoins.

Les $\mathrm{pH} 24$ des animaux ayant subi le traitement $\mathrm{n}^{\circ} 2$ sont particulièrement élevés puisqu'ils sont dans l'ensemble supérieurs à 6,00 . Bien que très significativement différentes de celles des lots témoins correspondants, les valeurs des pH 24 des lots 3 et 4 sont moins élevées que celles du lot 2 puisqu'elles se situent dans la zone 5,80-5,9o. La comparaison des valeurs des $\mathrm{pH} 45$ et des $\mathrm{pH} 24$ montre, en ce qui concerne les animaux traités, que dans le cas du traitement $n^{0} 2$, celles-ci sont très voisines, alors que dans le cas des traitements $n^{\circ} 3$ et $n^{\circ} 4$, les valeurs des $\mathrm{pH} 24$ sont notablement plus faibles que celles du $\mathrm{pH} 45$.

Ces différents résultats semblent indiquer que :

I. L'excitation immédiatement avant l'abattage ne se traduit pas, dans nos conditions expérimentales, par une chute de $\mathrm{pH}$ plus rapide que celle qui se produit dans les muscles d'animaux abattus en évitant toute excitation préalable.

Il convient toutefois de remarquer que les valeurs des $\mathrm{pH} 45$ des animaux témoins sont, dans l'ensemble, faibles. Il se pourrait que le mode d'abattage utilisé dans cette expérience soit responsable de cette chute rapide de $\mathrm{pH}$. Les convulsions suivant l'abattage seraient en effet susceptibles d'expliquer une accélération de la glycogénolyse.

2. L'excitation de l'animal 30 minutes avant 1'abattage peut provoquer une déplétion du glycogène musculaire. Il en résulte des $\mathrm{pH}$ peu compatibles avec une bonne conservation de la viande.

3. L'excitation 8 heures ou I 6 heures avant l'abattage conduit à des valeurs de $\mathrm{pH}$ très satisfaisantes. Une resynthèse de glycogène musculaire à partir du glucose sanguin et du glycogène hépatique peut expliquer les valeurs plus basses du $\mathrm{pH} 24$ dans les lots 3 et 4 que dans le lot 2. Selon WiSMER-PEDERSEN (I959), chez le porc le délai intervenant entre l'absorption de glucose et l'augmentation de teneur du muscle en glycogène serait en effet de l'ordre de 8 heures.

\section{Pouvoir de rétention d'eau}

Les résultats figurant sur le tableau $n^{\circ} 3$ montrent que les traitements $\mathrm{n}^{\mathrm{os}} 2$, 3 et 4 ont pour effet d'augmenter significativement le [pouvoir de rétention d'eau du tissu musculaire, ce qui était prévisible, compte tenu de la liaison existant entre le $\mathrm{pH}$ et la capacité de rétention d'eau (НАмм, I956).

\section{Couleur}

I1 ressort du tableau $n^{\circ} 4$ que le traitement I ne semble pas affecter 1'intensité de la coloration du tissu musculaire. Par contre, les traitements 2, 3 et 4 provoquent une augmentation de l'intensité de la coloration. Ces modifications de coloration sont imputables aux variations de $\mathrm{pH}$, puisqu'à teneur en pigment équivalente, une viande à haut $\mathrm{pH}$ paraît plus foncée qu'une viande à bas $\mathrm{pH}$ (WINKLER, I939). 

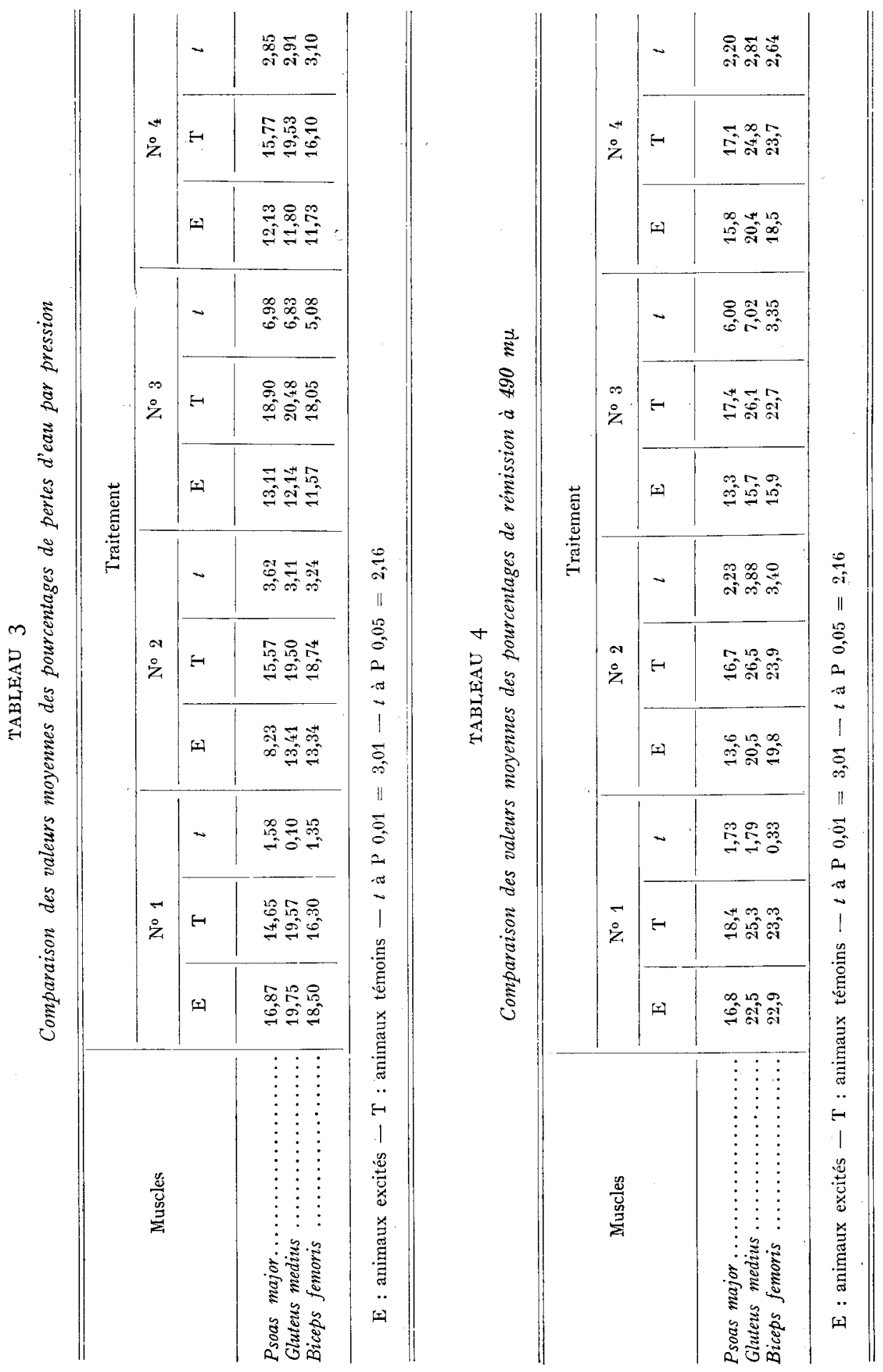


\section{III. - CONCLUSION}

Chez le porc, les conséquences d'une excitation ante mortem sur la qualité de la viande sont fonction du délai s'écoulant entre cette excitation et l'abattage.L'excitation immédiatement avant l'abattage ne semble pas, dans nos conditions expérimentales du moins, modifier les caractéristiques physico-chimiques du tissu musculaire que nous avons considérées dans cette étude, à savoir le $\mathrm{pH}$ " 45 minutes", le $\mathrm{pH}$ " 24 heures ", la capacité de rétention d'eau et l'intensité de la coloration. I apparaît, par contre, qu'un délai de 30 minutes intervenant entre l'excitation et l'abattage permet d'obtenir un $\mathrm{pH}$ musculaire élevé et, par suite, une amélioration de la rétention d'eatu.

Si l'excitation a lieu entre 8 et $\mathbf{I} 6$ heures avant l'abattage, les valeurs du pH ultime sont moins élevées que dans le cas précédent, ce qui pourrait vraisemblablement s'expliquer par une reconstitution du glycogène musculaire, à partir du glucose sanguin et du glycogène hépatique. Cette expérience montre donc qu'une excitation d'intensité relativement modeste, telle que celle qui se produit inévitablement lors du transport des animaux, peut avoir des répercussions non négligeables sur la qualité de la viande.

Reçu pour publication en juillet 1966.

\section{REMERCIEMENTS}

Nous tenons à remercier nos collègues de la Station de Recherches sur l'Élevage des Porcs qui ont eu l'obligeance de mettre à notre disposition les animaux dont nous avions besoin pour la réalisation de cette expérience.

\section{SUMMARY}

EFFECT OF EXCITEMENT BEFORE SLAUGHTER OF PIGS

ON SOME PHYSICO-CHEMICAL CHARACTERISTICS OF MUSCLE

Four groups of 8 Large White pigs of $100 \pm 5 \mathrm{~kg}$ liveweight were excited 5 or 30 minutes or 8 to 16 hours before slaughter. The excitement was application of 20 charges from an electric rod of a type currently in use in movement of livestock, which gives an electrical discharges every I 5 seconds. The pigs in these groups were compared with the same number of control pigs killed in their pens and for which care had been taken to avoid any previous excitement. All the animals were killed with a bolt humane killer. The carcases were put in a store at $+4^{\circ} \mathrm{C} 2$ hours after slaughter. The next day $\mathrm{pH}$, percentage reflectance at $490 \mathrm{my}$, and waterbinding capacity were estimated in psoas major, gluteus medius and biceps femoris muscles.

Results showed that in the conditions of the experiment excitement immediately before slaughter had no effect on the characteristics studied. Excitement 30 minutes before slaughter, on the other hand, increased $\mathrm{pH}$ colours and retention of water. The increases were less when the delay between excitement and slaughter was 8 or 16 hours.

The experiment shows that relatively mild excitement, but frequent during transport of animals, can have a marked effect on quality of the meat. 


\section{RÉFÉRENCES BIBLIOGRAPHIQUES}

Briskey F. J., ig64. Pale, soft, exudative porcine musculature. Adv. Food Res., 13, 89-1 78.

Callow E. H., 1936. The electrical resistance of muscular tissue and its relation to curing. Annu. Rep. Food Invest, Board, p. 57.

Callow E. H., I 937 a. The electrical resistance of muscular tissue and its relation to curing. Annu. Rep. Food Invest. Board, p. 75 .

Callow E. H., I937 b. Transport by rail and its after-effects on pigs. Annu. Rep. Food Invest. Board, p. 8I .

Callow E. H., 1938. Muscular fatigue and pH. Anmu. Rep. Food Invest. Board, p. 53 .

Callow E. H., 1939. The pH of muscular tissue. Annu. Rep. Food Inzest. Board, p. 27.

Hamm R., I956. Über die Wirkung der Adenosintriphosphor ... auf Hydratation und Rigidität des postmortalen Rindermuskels. Biochem. Z., 382, 309.

Henry M., Romani J. D., Joubert L., I958. La myopathie exsudative et dépigmentaire du porc. Maladie de l'adaptation. Essai pathogénique et conséquences pratiques, Rev. Palhol. gén. Physiol. clin., 696, 355-395.

Henry M., Billon J., 1959. Nouvelles observations sur l'influence des agressions non spécifiques sur la qualité de la viande de porc. Fifth Meat Res. Instit. Paris.

Lewis P. K. J., Brown C. J., Heck M. C., 1959. The effect of periodic electric shock prior to slaughter on the eating quality of fresh and cured pork. J. anim. Sci., 18, I477.

Lewis P. K. J., Brown C. J., HeCK M. C., r962. Effect of stress prior to slaughter on the chemical composition of raw and cooked pork. Food Sci., 27, 407 .

Sayre R. N., Briskey E. J., Heksstra W. G., Bray R. W., 196r. Effect of preslaughter change to a cold environment on characteristics of pork muscle. J. anim. Sci., 20, 487 .

SAYRE R. N., BRISKEY E. J., I963. Protein solubility as influenced by physiological conditions in the muscle. J. Food Sci., 28, 675.

WinkLer C. A., I939. Colour of meat. I. Apparatus for its measurement and relation between $\mathrm{pH}$ and colour. Canad. J. Res., 17, I-7.

Wismer-Pedersen J., 1959. Quality of pork in relation to rate of pH change post mortem. J. Food Sci., 24, 7 II.

Wismer-Pedersen J., Rieman H., 1960. Preslaughter treatment of pigs as it influences meat quality and stability. Res Cont. Am. Meat Inst. Found, 12th Conf. 